\title{
Patrones de aprendizaje en el contexto universitario mexicano: revisión sistemática del modelo y de sus publicaciones
}

Learning Patterns in the Mexican University Context: A Systematic Review of the Model and Publications Dealing with it

Padrões de aprendizagem no contexto universitário mexicano: revisão sistemática do modelo e suas publicações

\section{Laura B. García-Ravidá* (iD) orcid.org/0000-0002-3404-2829 \\ Lilia González Velázquez** (iD) orcid.org/0000-0001-9363-8684}

\section{(c) $\underset{\mathrm{BY}}{\mathrm{B}}(\mathrm{NC}$}

* Doctora en Psicología de la Educación. Profesora asociada. Departamento Psicología Básica, Evolutiva y de la Educación. Universitat Autònoma de Barcelona, España. Correo electrónico: laurabetiana.garcia@uab.cat

** Doctora en Filosofía y Ciencias de la Educación. Profesora investigadora a tiempo completo. Universitat Autónoma de Chiapas, México. Correo electrónico: lilglez@gmail.com 


\section{Resumen}

El objetivo del presente estudio de revisión es indagar la calidad y cantidad de investigaciones, con divulgación científica de impacto, sobre el modelo teórico-instrumental de patrones de aprendizaje propuesto por Vermunt y/o de aquellas que abordan uno o varios componentes que definen dicho modelo (concepciones de aprendizaje, orientaciones motivacionales, estrategias de procesamiento y de regulación) en el contexto universitario mexicano. Para ello, se realizó una búsqueda sistemática de publicaciones en revistas científicas indexadas según su impacto, mediante la selección de palabras clave, parámetro temporal y agrupación de acuerdo con las bases de datos, los autores y la indexación correspondiente. De acuerdo con los resultados, los investigadores reportaron una escasa cantidad de investigaciones que aplican el modelo de patrones de aprendizaje, así como un limitado número de publicaciones indexadas en Social Science Citation Index y Scopus. Finalmente, se concluye la necesidad de mejorar la calidad y cantidad de investigaciones que se llevan a cabo en el territorio mexicano en particular, y latinoamericano en general. Asimismo, se pide incrementar la divulgación de los resultados en inglés.

\section{Palabras clave}

Estilos de aprendizaje; estrategias de aprendizaje; motivación; revisión sistemática; discusión intercultural

\section{Keywords}

Learning style; learning strategies; motivation; systematic review; cross cultural debate
The aim of this review study is to look into the quality and quantity of research on Vermunt's theoretical and instrumental learning patterns model-with an impact from the perspective of scientific dissemination-and/or those dealing with one or more of its components (learning conceptions, motivational orientations processing and regulation strategies) in the Mexican context. For this purpose, the researchers carried out a systematic search of publications in indexed journals according to their impact, keywords, a time parameter and their association according to the databases, the authors and their corresponding indexation. The results showed a small amount of research applying the learning patterns model, as well as a limited number of indexed publications in the Social Science Citation Index and Scopus. Finally, the authors concluded that it is necessary to improve the quality and quantity of research carried out in the Mexican territory, in particular, and Latin American in general. Besides, they asked for enhancing the dissemination of results in English

\section{Resumo}

O objetivo deste estudo de revisão é investigar a qualidade e quantidade de pesquisas, com divulgação científica do impacto, sobre o modelo teórico-instrumental de padrões de aprendizagem proposto por Vermunt e / ou aqueles que abordam um ou vários componentes que definem o referido modelo (concepções de aprendizagem, orientações motivacionais, estratégias de processamento e regulação) no contexto universitário mexicano. Para isso, foi realizada uma busca sistemática de publicações em periódicos científicos indexados de acordo com o seu impacto, selecionando palavras-chave, parâmetros temporais e agrupamentos de acordo com as bases de dados, autores e a indexação correspondente. De acordo com os resultados, os pesquisadores relataram uma pequena quantidade de pesquisa que aplica o modelo de padrão de aprendizagem, bem como um número limitado de publicações indexadas no Social Science Citation Index e no Scopus. Finalmente, conclui-se a necessidade de melhorar a qualidade e a quantidade de pesquisas realizadas no território mexicano em particular, e na América Latina em geral. Da N. ${ }^{\circ} 77$ mesma forma, solicita-se aumentar a divulgação dos resultados em inglês.

\section{Palavras-chave}

estilos de aprendizagem; estratégias de aprendizagem; motivação; revisão sistemática; discussão intercultural 


\section{Introducción}

Uno de los grandes consensos desde las dos últimas décadas del siglo xx es que la educación formal debe transformarse profundamente. Son muchas las razones para esta urgencia; pero, sin duda, una que tiene especial importancia es la persistencia en la escuela del paradigma educativo conocido como tradicionalista, en el cual el docente posee el conocimiento y el alumno lo recibe pasivamente y lo reproduce. Las consecuencias de estas prácticas pedagógicas en el desempeño académico de los estudiantes y en la calidad de sus aprendizajes han sido objeto de estudio de numerosas investigaciones realizadas desde diversas disciplinas, teorías y enfoques, tales como la psicología cognitiva, educativa y de la personalidad, la filosofía, la sociología, la antropología y las neurociencias, entre otras. De esta manera, podemos señalar que a partir de la década de los ochenta se ha ido profundizando en ciertas particularidades, y combinación de variables, que definen determinadas características del aprendizaje. Así, autores como Säljö (1979), Marton, Dall' Alba y Beaty (1993) indagaron acerca de qué concepciones definen el aprendizaje, mientras que otros, como Deci (1972), Weiner (1979, 1983), Pintrich et al. (1988) y Deci y Ryan (1985) profundizaron en la influencia de la motivación en el proceso de aprendizaje de los estudiantes. Asimismo, otros autores se centraron en analizar cómo las estrategias de procesamiento (Beltrán, 1993; Entwistle, 1988; 1991; Weinstein y Mayer, 1986) y de regulación (Pintrich, 1994; Pintrich, Smith, García y McKeachie, 1993; Pintrich y De Groot, 1990; Zimmerman, 1989, 1990) caracterizan a ciertos perfiles de estudiantes universitarios (tabla 1). Si bien todos ellos remarcaron la relevancia de dichos aspectos, lo que abrió nuevos horizontes a la investigación en educación, solo fue a mediados de los noventa cuando Jan Vermunt dio un paso más allá y propuso un modelo que integra los componentes citados (concepciones de aprendizaje, orientaciones motivacionales, estrategias de procesamiento y de regulación) con el objetivo de identificar cuatro patrones de aprendizaje (Vermunt, 1996, 1998).

\section{Patrones de aprendizaje}

Los patrones de aprendizaje propuestos por Vermunt (1998) son el resultado de la combinación de cuatro componentes (concepciones de aprendizaje, orientaciones motivacionales, estrategias de procesamiento y de regulación), que fundamentan un modelo integrador. De esta manera, el autor propone cuatro patrones de aprendizaje: dirigido al significado (MD), dirigido a la aplicación (AD), dirigido a la reproducción (RD) y no dirigido (UD) (tabla 2). 


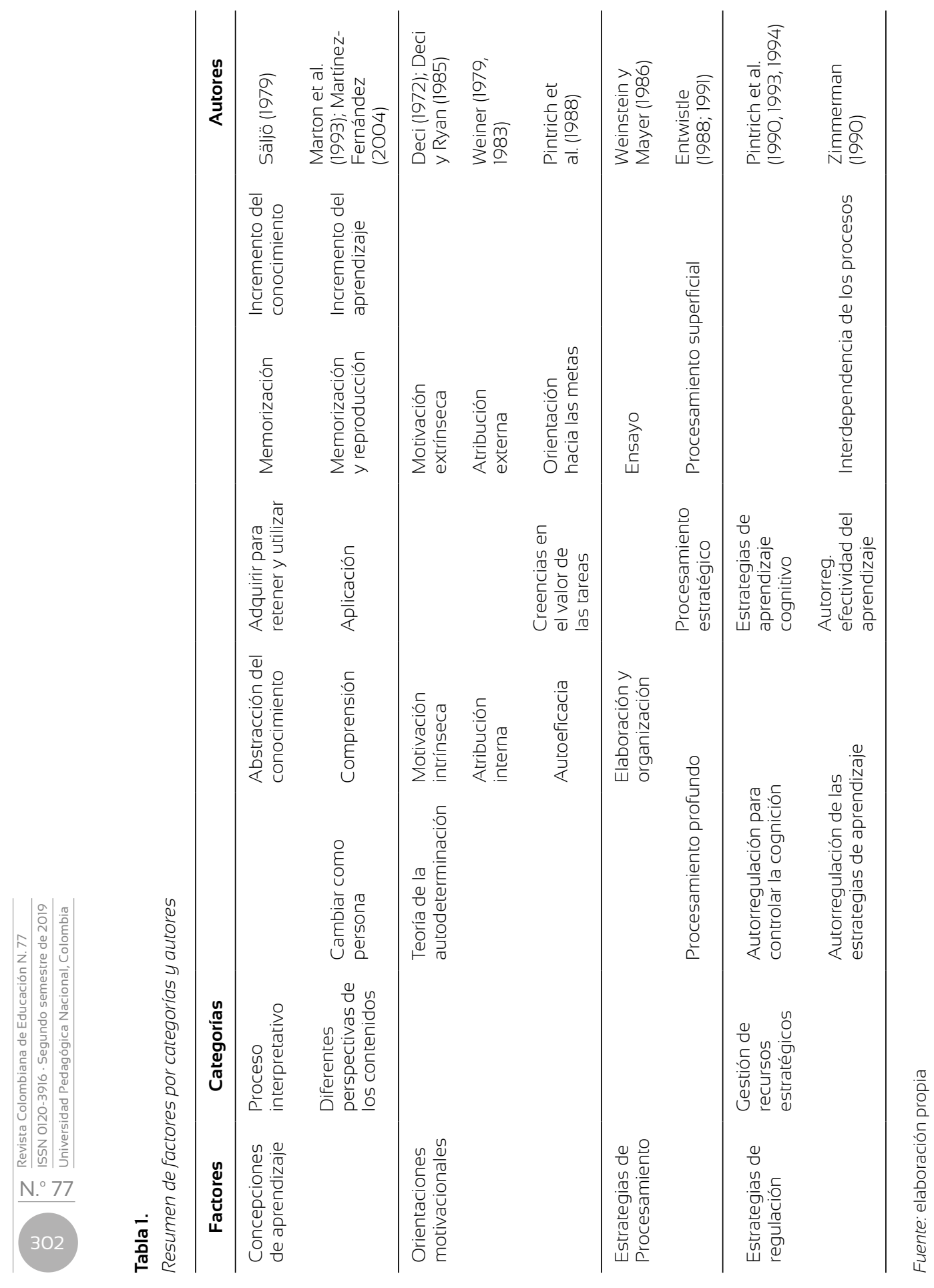



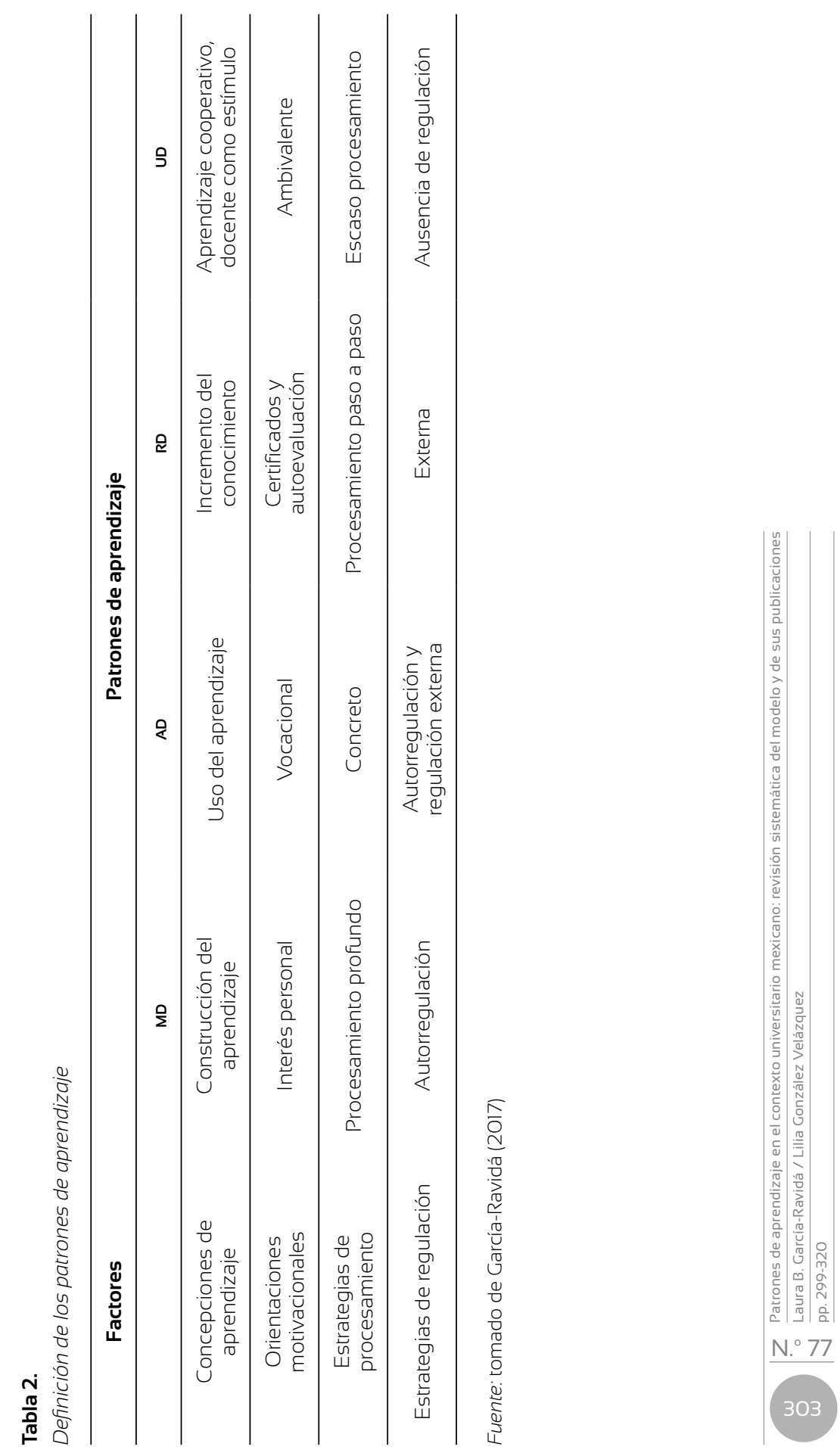
Durante el periodo de 1998 a 2009 el modelo se fue validando y fortaleciendo en el marco del territorio noreuropeo, en países como Bélgica (Donche y Van Petegem, 2009; Ferla, Valcke y Schuyten, 2008); Holanda (Busato, Prins, Elshout y Hamaker, 1998; Veenman, Prins y Verheij, 2003; Vermunt, 1998, 2005; Vermunt y Minnaert, 2003) y Reino Unido (Boyle, Duffy y Dunleavy, 2003). A finales de los años noventa, se aplicó a estudiantes de Indonesia (Ajisuksmo y Vermunt, 1999), y alcanzó mayor relevancia en el territorio oriental a finales de la década de los 2000. Así, el constructo teórico se expandió por Australia (Smith, Krass, Sainsbury y Grenville., 2010), China (Biemans y Van Mil, 2008), Turquía (Kalaca y Gulpinar, 2011) y Sri Lanka (Marambe, Athuraliya, Vermunt y Boshuizen, 2007). De esta manera, se puede observar cómo el modelo ha ido migrando y validándose en diversos contextos y culturas.

En lo que respecta al territorio iberoamericano, si bien el modelo de patrones de aprendizaje comenzó a divulgarse a mediados de la primera década del siglo xxı (Argentina: Alves de Lima et al., 2006), solo en la década del 2010 se ha profundizado en su análisis. Así, la investigación se ha centrado, desde Latinoamérica, en Argentina (De la Barrera, Donolo y Rinaudo, 2010; González y Difabio de Anglat, 2016; Vázquez, 2009); Colombia, México y Venezuela (García-Ravidá, 2017; Martínez-Fernández y Vermunt, 2015). En lo que respecta al sur de Europa, las investigaciones se han focalizado en España (Martínez-Fernández y García-Ravidá, 2012; Martínez-Fernández y Vermunt, 2015) y Portugal (Rocha y Ventura, 2011).

De manera particular, en la presente investigación se busca conocer la situación de México como un ejemplo de los países latinoamericanos donde existe una creciente y diversa población de estudiantes universitarios que enfrenta situaciones sociales complejas como la pobreza, la violencia y el desempleo, pero también que tiene aspiraciones de movilidad social, resiliencia y compromiso colectivo. Por otra parte, si bien en este país se ha incrementado la cobertura de la educación obligatoria de manera significativa, hay cuestionamientos respecto a su calidad (Castañeda Figueiras y Peñalosa, 2016). En el caso de la educación preuniversitaria, durante los últimos quince años el país ha quedado en las últimas posiciones en los resultados de evaluaciones internacionales como la Prueba PISA, que evalúa habilidades de razonamiento y solución de problemas aplicados a la lectura, las matemáticas y las ciencias (Márquez, 2017). Por lo tanto, en este artículo se explora cómo estudian y conciben el aprendizaje los estudiantes mexicanos y cuáles son sus motivaciones y estrategias para alcanzar sus metas. 
De esta manera, el principal objetivo es revisar las investigaciones que se han reportado sobre dichos patrones, o de los componentes que los definen, en este caso en el ámbito de la educación superior mexicana. Todo ello, con el propósito de obtener un primer mapa del estado de la cuestión en el contexto mexicano.

Así, las preguntas de investigación son:

» ¿Cuáles estudios han abordado alguno de los componentes que definen el modelo de patrones de aprendizaje en el ámbito universitario mexicano? ¿Qué instrumentos han aplicado?

¿QQué investigaciones sobre patrones de aprendizaje se han llevado a cabo aplicando el modelo e instrumento propuesto por Vermunt?

¿ ¿Cuál es la calidad y cantidad de publicaciones realizadas en el contexto del territorio mexicano?

\section{Metodología}

Con el objetivo de llevar a cabo una estimación del estado del arte y de la calidad de las investigaciones acerca de los patrones de aprendizaje y/o alguno de sus componentes en el ámbito de la educación superior en el territorio mexicano, se realizó una búsqueda bibliográfica en cuatro pasos. Así, el procedimiento consistió en:

1. Selección de palabras clave: estas responden a cada uno de los componentes que definen los patrones de aprendizaje.

2. Selección del parámetro temporal: se selecciona desde el año 2000 debido a que el modelo fue publicado entre 1996 y 1998.

3. Identificación de bases de datos, depósitos digitales de documentos o repositorios digitales y autores.

4. Caracterización de los estudios según su indexación en Social Science Citation Index y en Scopus (tabla 3).

Los puntos 3) y 4) se consideran los más oportunos para evaluar la calidad de las investigaciones, ya que son criterios consensuados internacionalmente. Asimismo, al tratarse de una revisión sistemática de investigaciones científicas (artículos, libros, tesis de posgrado) se han excluido aquellas difusiones en medios de divulgación masiva no científicas. 


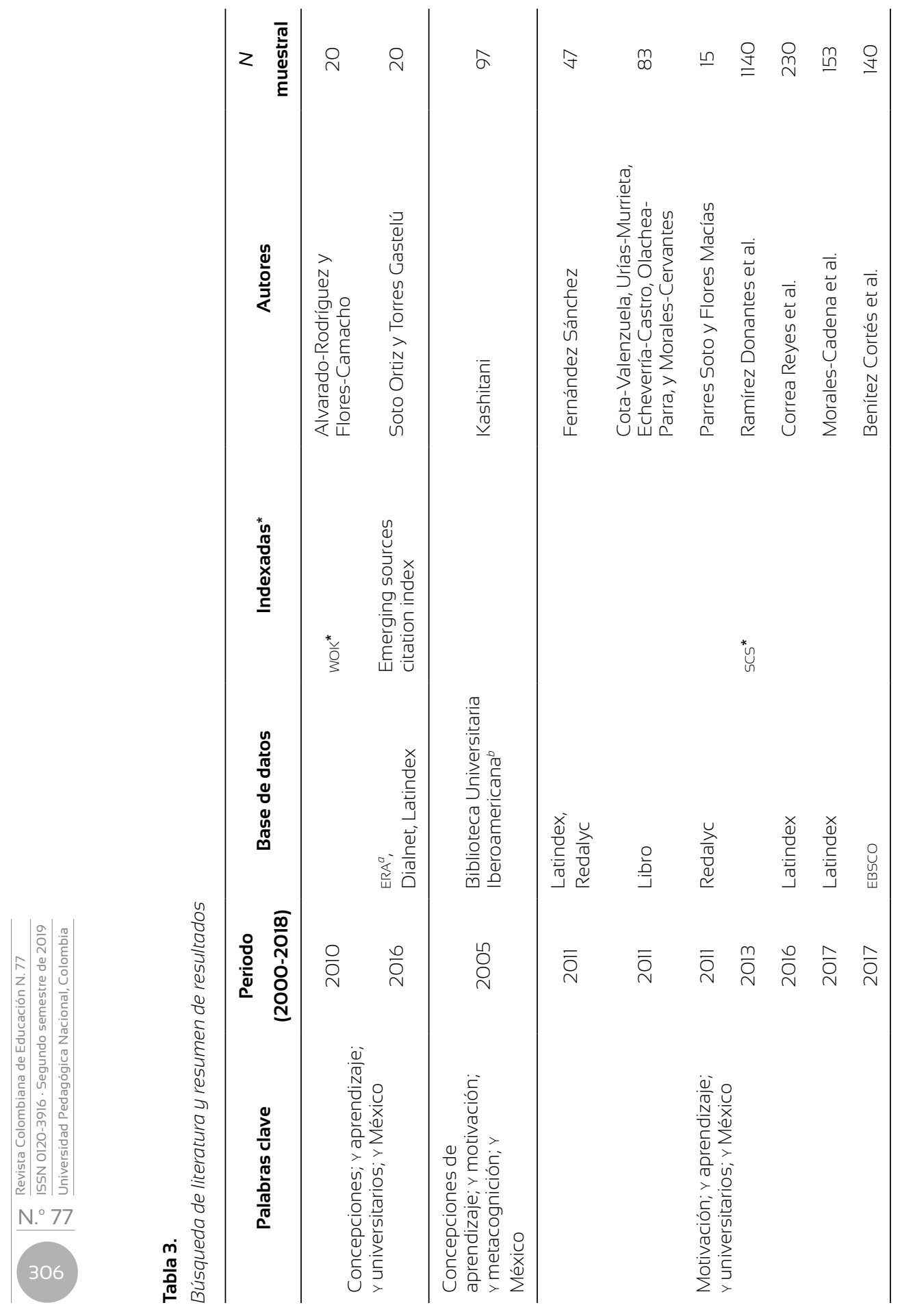



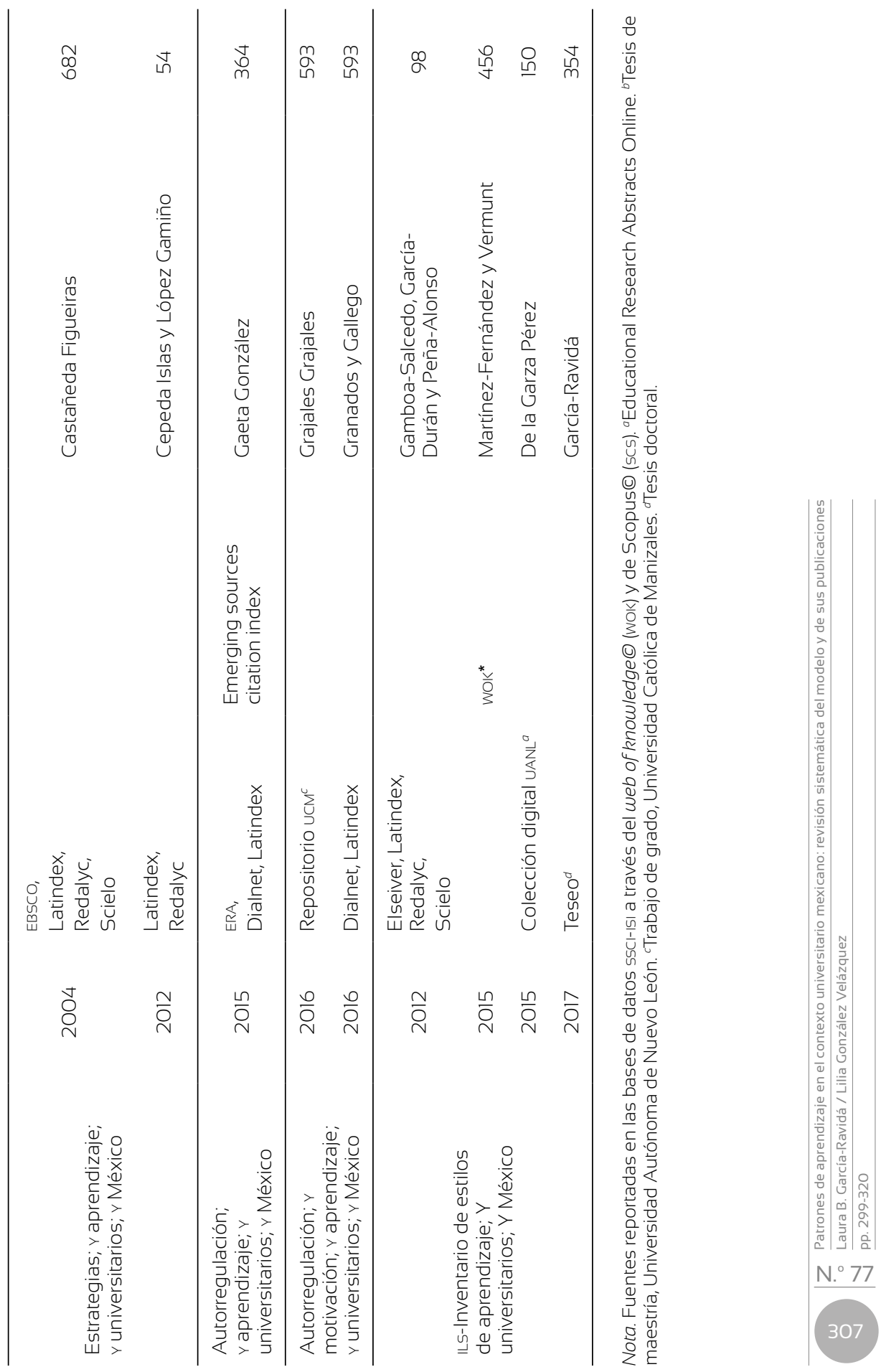


\section{Resultados}

Los resultados pueden agruparse en dos categorías, que responden a las dos primeras preguntas de investigación planteadas. La primera, reúne las investigaciones en las cuales se ha indagado acerca de alguno de los componentes que estructuran el modelo de Vermunt (concepciones de aprendizaje, orientaciones motivacionales, estrategias de aprendizaje y de regulación) y que han aplicado diversos cuestionarios. La segunda, se centra en las investigaciones en las que se ha aplicado y analizado el modelo de patrones de aprendizaje (Vermunt, 1998) y el cuestionario que los identifica (Inventory of Learning Styles- en inglés, ILS). Cabe resaltar que en el apartado de Referencias se agrupará la bibliografía manteniendo esta distinción.

Con relación a la primera categoría, se han hallado tres artículos que investigan las concepciones de aprendizaje. La primera investigación indaga acerca de las concepciones de los investigadores universitarios (Alvarado-Rodríguez y Flores-Camacho, 2010). Para ello diseñaron una guía de entrevista extensa que se aplicó a veinte investigadores de diversos centros científicos (biología, ciencias de la atmósfera, nucleares, astronomía, ecología, matemáticas, química y física). Los resultados pusieron en evidencia las propuestas y/o su justificación. Así, se destacaron los siguientes aspectos: 1) El estudiante es el único responsable de su proceso de aprendizaje, 2) deben relacionarse las actividades cotidianas con la teoría del aula, 39 la calidad de la enseñanza es proporcional a la formación del docente, 4) la mejor vía de enseñanza de la ciencia es la experimentación, y 5) los estudiantes necesitan estar motivados.

El segundo artículo, de Soto Ortiz y Torres Gastelú (2016), propone un esbozo del perfil universitario a partir de la percepción y las expectativas de veinte estudiantes de Ciencias Sociales y Administrativas, que fueron entrevistados por medio de grupos focales. Los autores concluyeron que el estilo de los estudiantes se caracteriza por ser arcaico, ya que se centra en concepciones memorísticas orientadas a la superación de las asignaturas. De igual manera, los universitarios resaltan la concordancia entre un desarrollo interpersonal y las conductas (positivas o negativas) de los docentes; como consecuencia vinculan su aprendizaje a la figura del profesor-tutor como guía actitudinal y motivacional del aprendizaje.

Por su parte, Kashitani (2005), en su tesis de maestría, indaga acerca de las concepciones de aprendizaje y enseñanza de 97 docentes universitarios provenientes de diversos dominios específicos (Ciencias Exactas, Sociales y Humanidades); orientaciones motivacionales y estrategias metacognitivas de los estudiantes según la apreciación de sus docentes; para 
ello empleó un cuestionario de elaboración propia. Entre los resultados cabe destacar como concepciones a promover por parte de los docentes la aplicación de los conocimientos, la inquietud para investigar, la motivación para el aprendizaje y la creatividad. En sentido opuesto, los aspectos menos relevantes por considerar fueron la memorización, la puntualidad y seguir las instrucciones de los profesores. A su vez, cerca de la mitad de los docentes señaló que muy pocos estudiantes o ninguno tiene una buena capacidad de comunicación oral y escrita, ni disponen o aplican estrategias metacognitivas efectivas. En cambio, el $50 \%$ de los docentes opinó que casi todos los estudiantes tienen confianza en sí mismos, son responsables y atentos a las instrucciones recibidas. Respecto a las características positivas que definen a un buen docente universitario se destaca la importancia de la actualización de los conocimientos de cada campo, vocación por la profesión y, en menor medida, facilitar que el estudiante aprenda a aprender. Asimismo, los aspectos menos relevantes han sido hacer un seguimiento de las metas de aprendizaje, el manejo efectivo del grupo y de la disciplina en el aula.

Respecto a la motivación u orientaciones motivacionales se reportan siete investigaciones, en las cuales se ha utilizado principalmente el Cuestionario de Motivación y Estrategias de aprendizaje (MSLQ) de Pintrich, Smith, García y McKeachie (1991).

Fernández Sánchez (2011) realizó un estudio con 47 universitarios en el cual indagó acerca de los estilos y las motivaciones en la educación superior a partir del aprendizaje colaborativo. Los resultados señalaron que, luego de las intervenciones, las mujeres presentaron una disminución significativa en la escala de ansiedad; al mismo tiempo, los hombres mostraron una menor orientación extrínseca. En ambos casos, luego de la intervención aplicada los índices disminuyeron junto a la búsqueda de ayuda. Así, confirman la relevancia del trabajo cooperativo y el B-learning para disminuir los aspectos mencionados.

Cota-Valenzuela et al. (2011) realizaron un estudio descriptivo acerca del nivel de motivación y uso de estrategias de aprendizaje en 83 estudiantes de Ciencias de la Educación. En sus resultados reportaron que estos, en general, mostraron una motivación y un uso de estrategias de aprendizaje superiores a la media teórica de la versión en castellano del instrumento (Sotelo, 2007).

Parres Soto y Flores Macías (2011) trabajaron con 15 estudiantes del área de Ciencias Sociales y Humanidades en un taller de arte visual para evaluar su motivación y estrategias de aprendizaje. Los resultados indicaron diferencias significativas a favor del incremento de la motivación, la autorregulación y la búsqueda de ayuda luego del taller. 
Ramírez Dorantes et al. (2013) tradujeron, adaptaron y validaron el cuestionario MSLQ al contexto mexicano a partir de una muestra de 1140 universitarios de 12 titulaciones diferentes. Para la versión definitiva del instrumento se conservó la estructura teórica original y se elaboraron cuadernillos con los reactivos traducidos y hojas de respuestas.

Correa Reyes, Cuevas Martínez y Villaseñor Ponce (2016) indagaron acerca de las diferencias entre el bienestar psicológico, las metas y el rendimiento académico en 230 universitarios. De esta manera, hallaron que los estudiantes con mejor rendimiento académico presentaban relaciones positivas con el bienestar psicológico y las metas. En sentido contrario, los estudiantes con menor rendimiento mostraron una mayor orientación a la meta extrínseca.

Morales et al. (2017) investigaron sobre la importancia de la motivación y las estrategias de aprendizaje en la enseñanza de la medicina. El estudio se realizó con 153 universitarios de la carrera de Medicina; los resultados señalaron que los que contaban con mayor tiempo para realizar las tareas, tenían menos responsabilidades y disponían constantemente de actividades novedosas mostraban mayor motivación intrínseca. Por su parte, los estudiantes de inicio de carrera mostraban mayor ansiedad que el resto de sus pares. Por último, los residentes de posgrado mostraron mayor motivación intrínseca, mayor esfuerzo, pero menor organización que sus pares de pregrado.

Benítez Cortés et al. (2017) analizaron la percepción de 140 alumnos acerca de la instrucción, las actividades y el apoyo que reciben de sus docentes. Así, los estudiantes valoran positivamente para su aprendizaje una secuencia ordenada de actividades, estrategias enfocadas en la práctica, el uso de medios digitales y el apoyo constante que recibieron por parte de los profesores.

Con relación a las estrategias de aprendizaje, se destacan los trabajos de Castañeda Figueiras (2004), quien evaluó y fomentó la investigación acerca del desarrollo cognitivo y el aprendizaje complejo. Todo ello, a partir de la evaluación enmarcada en un trabajo multicomponencial de medición de resultados de aprendizaje, en la que participaron 682 estudiantes de psicología. En el mismo estudio, la autora presenta un desarrollo tecnológico con base en el marco multidimensional, a partir del Inventario de estilos de aprendizaje y Orientación motivacional al estudio, ambos centrados en la ejecución del aprendizaje y en su evaluación. El estudio fue validado en 5000 estudiantes universitarios de todo el país. De ello, se desprende un programa de enseñanza y aprendizaje estratégicos, ampliamente difundido por la autora. 
Por su parte, Cepeda Islas y López Gamiño (2012) emplearon la Escala de estrategias de aprendizaje (ACRA, tercera edición) en 54 estudiantes de psicología, con el objetivo de evaluar sus estrategias de aprendizaje y sus habilidades verbales. De acuerdo con los resultados, los estudiantes noveles muestran ciertas deficiencias en el dominio de estrategias y habilidades verbales, lo cual se explica porque utilizan dichas estrategias para el logro de un conocimiento a corto plazo, sin considerar que el aprendizaje debe ser a largo plazo.

Con respecto a la autorregulación, Gaeta González (2015) empleó el Cuestionario para la evaluación de metas académicas (CEMA) y la Escala de evaluación de la autorregulación del aprendizaje a partir de textos (Aratex), con el objetivo de evaluar qué aspectos personales favorecen la autorregulación en la comprensión de textos académicos. La investigación contó con la participación de 364 estudiantes de ingeniería. Los resultados evidenciaron la influencia de los conocimientos previos y de metas claras para favorecer el uso de estrategias de autorregulación en la comprensión de textos académicos.

En esta línea de investigación otros autores analizaron las relaciones entre la autorregulación y las motivaciones; para ello emplearon el Cuestionario de motivación y estrategias de aprendizaje CMEA traducción mexicana del mSLQ (Grajales Grajales, 2016; Granados y Gallego, 2016).

De este modo, Grajales Grajales (2016) señaló, en su estudio con 593 universitarios, que la autorregulación metacognitiva es un factor determinante en la asociación de estrategias cognitivas como la repetición, la elaboración y la organización.

Por su parte, Granados y Gallego (2016) examinaron la relación entre el aprendizaje autorregulado, las estrategias de aprendizaje y la motivación intrínseca de 593 estudiantes de las carreras de Ciencias Médicas, Lenguas Modernas y Educación. Sus resultados mostraron una relación positiva entre las variables.

Respecto a la segunda categoría, esto es, los estudios que han aplicado el modelo de patrones de aprendizaje de Vermunt, solo se hallaron dos artículos publicados (Gamboa-Salcedo, García-Durán y Peña-Alonso, 2012; Martínez-Fernández y Vermunt, 2015), una tesis de maestría (De la Garza Pérez, 2015) y una tesis de doctorado (García-Ravidá, 2017).

En el primer artículo se realiza la traducción al español del Inventario de estilos de aprendizaje y se analiza su confiabilidad en 98 residentes de pediatría. Dicha traducción mantuvo la estructura de 120 ítems y arrojó una fiabilidad de 0,91, por lo que se logró una adecuada adaptación contextual. En este sentido, se señala la importancia de la traducción y la 
adaptación cultural de los instrumentos como condición básica para que el intercambio de herramientas y su posterior aplicación contribuyan a la discusión acerca de la globalización educativa.

En el segundo artículo, Martínez-Fernández y Vermunt (2015) realizaron la traducción, adaptación y validación del instrumento contando con 456 universitarios latinoamericanos, de los cuales 100 eran provenientes de México. Los autores mantuvieron los 120 ítems del instrumento original y reportaron adecuados índices de fiabilidad ( 0.57 a 0.88 ), salvo en la escala de interés personal que ha debido ser adaptada, conceptualmente, al contexto iberoamericano. De esta manera, los autores hallaron una estructura de cuatro patrones de aprendizaje, más similar a las halladas en estudiantes asiáticos que la reportada en el modelo original. Encontraron que las muestras provenientes de México y España se inclinaban, en mayor medida, hacia una visión pasiva de los docentes y una instrucción más individual que sus pares de Colombia y Venezuela. Por último, señalan una vía de activación del procesamiento profundo por medio de la construcción del conocimiento y de la regulación externa con cierto grado de autorregulación. Todo ello, ha dado lugar a la denominada paradoja latinoamericana y española.

Por su parte, De la Garza Pérez (2015) buscó identificar los patrones de aprendizaje en 150 estudiantes de psicología. Al contrario del modelo original, que propone cuatro patrones de aprendizaje, el autor identificó cinco:

1. Patrón de aprendizaje dirigido al significado y a la aplicación

2. Patrón pasivo idealista (patrón definido a partir del predominio de las concepciones de aprendizaje)

3. Patrón no dirigido

4. Patrón dirigido a la reproducción con vocación

5. Patrón pasivo motivacional (patrón definido por la preponderancia de las orientaciones motivacionales).

El autor no halló diferencias de género ni de edad en relación con el patrón pasivo motivacional.

Finalmente, García-Ravidá (2017), en su tesis doctoral, exploró y discutió el modelo de patrones de aprendizaje y su relación con ciertos factores personales y el rendimiento académico en universitarios latinoamericanos; del total de 354 participantes 100 eran estudiantes mexicanos de pedagogía. En sus resultados, identificó la misma estructura de cuatro patrones de aprendizaje propuesto por Vermunt, pero con ciertos matices propios del contexto iberoamericano (Vermunt, Bronkhorst y Martínez-Fernández, 2014). Así, los patrones hallados se denominaron patrón 
de aprendizaje dirigido al significado con regulación externa; patrón pasivo-idealista; patrón dirigido a la reproducción; y patrón lack of (carente de regulación). Asimismo, reportó que los varones se identificaban con el cuarto patrón; solo aquellos que se dedicaban exclusivamente a sus estudios se identificaban con el primer patrón. En cambio, los que compartían sus estudios con otras actividades se identificaban con el segundo. Por último, identificó relaciones significativas entre el patrón dirigido al significado con regulación externa y un rendimiento académico exitoso.

En relación con la tercera pregunta, acerca de la calidad y la cantidad de publicaciones realizadas en el contexto de la educación superior en el territorio mexicano, en cuanto los patrones de aprendizaje o variables relacionadas con alguno de sus componentes, se identifican dos aspectos relevantes:

» Nivel de indexación de las publicaciones: de un total de 15 artículos solo 5 (33,3\%) se encuentran en revistas indexadas; dos de ellas son emergentes Los diez restantes $(66,7 \%)$ no se corresponden con altos estándares de calidad internacional. Del resto de publicaciones, 3 son tesis de maestría, una tesis doctoral y, por último, un capítulo de libro.

" Cantidad de publicaciones: la mayoría de las investigaciones (15) indagan acerca de $1 \mathrm{o}$, a lo sumo, combinan 2 componentes del aprendizaje; por ejemplo: concepciones de aprendizaje, motivación-estrategias de autorregulación, etc. En estas predomina el empleo de entrevistas y del cuestionario MSLQ. En cambio, solo cuatro analizan el modelo de patrones de aprendizaje, adaptando y aplicando el cuestionario ILS.

\section{Discusión}

El objetivo principal del presente estudio ha sido indagar acerca de la divulgación del modelo de patrones de aprendizaje (Vermunt, 1998) en el contexto de la educación superior en el territorio mexicano.

Con relación a la primera pregunta de investigación, acerca de cuáles estudios han investigado alguno de los componentes que definen el modelo de patrones de aprendizaje en el ámbito universitario mexicano y los instrumentos que han aplicado, se han identificado quince investigaciones que analizan algunos de los componentes que definen el modelo teórico de Vermunt. Algunos de ellos los analizan por separado y el resto examina la relación entre dos o tres de los cuatro. De esta manera, se muestran relaciones entre el aprendizaje superficial, el bajo rendimiento, concepciones memorísticas y la motivación externa (Alvarado-Rodríguez,

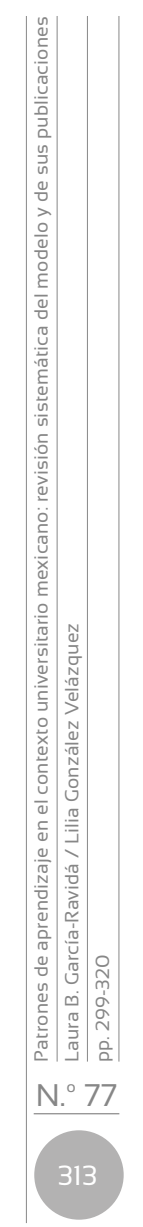


y Flores-Camacho, 2010; Soto Ortiz, y Torres Gastelú, 2016). Asimismo, se destaca la demanda de un rol activo por parte de los docentes y una constante orientación hacia los estudiantes (Benítez Cortés et al., 2017; Castañeda Figueiras y Peñalosa Castro, 2016; Kashitani, 2005). Por otro lado, se resaltan las escasas estrategias y habilidades verbales que presentan los estudiantes noveles (Cepeda Islas y López Gamiño, 2012), en oposición a la motivación intrínseca, mayor esfuerzo, autorregulación y metas claras que se encuentran en los estudiantes de cursos avanzados (Gaeta González, 2015; Morales-Cadena et al., 2017; Parres Soto y Flores Macías, 2011).

Respecto a la segunda pregunta, acerca de qué investigaciones sobre patrones de aprendizaje se han Ilevado a cabo aplicando el modelo teórico e instrumental propuesto por Vermunt, solo se han hallado dos artículos científicos que traducen al español y validan el cuestionario ILS. En ambos casos se obtienen datos fiables para su aplicación y se señala la necesidad de mejorar su adaptación contextual (Gamboa-Salcedo, García-Durán y Peña-Alonso, 2012; Martínez-Fernández y Vermunt, 2015). A su vez, se identifican una tesis de maestría y una doctoral que ponen de relieve los matices que aporta el territorio mexicano a la definición de los patrones de aprendizaje (De la Garza Pérez, 2015; García-Ravidá, 2017).

A partir de estos resultados es necesario destacar la coherencia y coincidencia de los hallazgos reportados en las investigaciones que analizan los componentes por separado y aquellos que han empleado el modelo integrador de Vermunt, en el contexto iberoamericano (Alves de Lima et al., 2006; De la Barrera, Donolo y Rinaudo, 2010; Martínez-Fernández y García-Ravidá, 2012, 2017; González y Difabio de Anglat, 2016; Vázquez, 2009; Martínez-Fernández y Vermunt, 2015; Rocha y Ventura, 2011). Por ello, se evidencia la necesidad de profundizar en la implementación del modelo, contextualizarlo y debatir sus puntos fuertes y débiles desde la perspectiva territorial.

A partir de la tercera pregunta, acerca de la calidad y cantidad de publicaciones realizadas en el contexto del territorio mexicano, se comenzó la búsqueda en revistas indexadas con alto índice de impacto (ssCl-Scopus). Fue grande la sorpresa al encontrar una escasa cantidad de publicaciones en poblaciones universitarias provenientes de México.

De esta manera, no solo se manifiesta la escasez de divulgación científica, la poca variedad en la elección de instrumentos de evaluación, sino también, la necesidad de contribuir con nuestros aportes, por ahora escasos, a la discusión intercultural en el ámbito internacional (Vermunt y Donche, 2017). Por ello, además de incrementar las investigaciones 
acerca de los patrones de aprendizaje en el territorio iberoamericano, y en el caso que nos ocupa, el contexto mexicano, es necesario identificar e integrar en dichas investigaciones variables sociodemográficas, emocionales y culturales, entre otras. Todo ello, con el propósito de comprender la diversidad que amplía las miradas aportando nuevas visiones que enriquezcan el modelo y contribuyan a la mejora de la educación, en cuanto a su calidad, equidad e inclusión en el ámbito internacional.

Por otro lado, estos resultados, en su mayoría, se hallan publicados en idioma español. Ello es importante para la divulgación dentro de la comunidad hispana, ya que difunde investigaciones de alto interés y rigurosidad; pero, al mismo tiempo, contribuye a mantener el vacío antes mencionado en cuanto a la presencia académica de Latinoamérica en el contexto internacional.

Por lo anterior, resulta imprescindible actuar sobre cuatro cuestiones básicas:

1. Profundizar en modelos integradores como la propuesta de patrones de aprendizaje.

2. Elevar la calidad de las publicaciones para acceder a revistas de alto índice de impacto.

3. Aumentar la cantidad de publicaciones en inglés, ya que es el idioma predominante en las discusiones interculturales en el plano internacional.

4. Incrementar la creación de redes entre investigadores e instituciones para colaborar en la prosecución de los tres ítems anteriores.

Definitivamente tenemos por delante el gran desafío de incluir la voz de los investigadores latinoamericanos en el plano del debate internacional.

\section{Referencias}

Ajisuksmo, C. y Vermunt, J. D. (1999). Learning styles and self-regulation of learning at university: An Indonesian study. Asia Pacific Journal of Education, 19(2), 45-59. DOI: 10.1080/0218879990190205.

Alvarado Rodríguez, M. E., y Flores-Camacho, F. (2010). Percepciones y supuestos sobre la enseñanza de la ciencia. Las concepciones de los investigadores universitarios. Perfiles Educativos, 32(128), 10-26.

Alves De Lima et al. (2006). Learning strategies used by cardiology residents: Assessment of learning styles and their correlations. Education for Health, 19(3), 289-297. DOI: 10.1080/13576280600937788.

Beltrán, J. (1993). Procesos, estrategias y técnicas de aprendizaje. Madrid: Síntesis. 
Benítez Cortés, R. P., Aguilar Navarrete, P., Camacho González, M. F. Y., y Torres Covarrubias, V. J. (2017). Medios instruccionales que apoyan el aprendizaje de la programación de computadoras en estudiantes universitarios de carreras de Tı. Revista Internacional de Educación en Ingeniería, 9(1), 1-7.

Biemans, H. y Van Mil, M. (2008). Learning styles of Chinese and Dutch students compared within the context of Dutch higher education in life sciences. The Journal of Agricultural Education and Extension, 14(3), 265-278. DOI: 10.1080/13892240802207700.

Boyle, E. A., Duffy, T. y Dunleavy, K. (2003). Learning styles and academic outcome: The validity and utility of Vermunt's Inventory of Learning Styles in a British higher education setting. British Journal of Educational Psychology, 73, 267-290. Dol: 10.1348/0070990360626976.

Busato, V. V., Prins, F. J., Elshout, J. J. y Hamaker, C. (1998). Learning styles: A cross-sectional and longitudinal study in higher education. British Journal of Educational Psychology, 68, 427-441. DOI:10.1111/j.2044-279.1998.tb01302.x.

Castañeda Figueiras, S. y Peñalosa Castro, E. (2016). Fenomenología de agencia académica. México: UAM.

Castañeda-Figueiras, S. (2004). Evaluando y fomentando el desarrollo cognitivo y el aprendizaje complejo. Psicología desde el Caribe, 13, 109-143.

Cepeda Islas, M. L. y López Gamiño, M. R. (2012). Evaluación de estrategias de aprendizaje y habilidad verbal en una muestra de estudiantes universitarios. Enseñanza e Investigación en Psicología, 17(1), 117-135.

Correa Reyes, A. S., Cuevas Martínez, M. D. R. y Villaseñor Ponce, M. (2016). Bienestar psicológico, metas y rendimiento académico. Vertientes. Revista Especializada en Ciencias de la Salud, 19(1), 29-34.

Cota-Valenzuela, L. V., Urías-Murrieta, M., Echeverría-Castro, S. B., Olachea-Parra, L. F. y Morales-Cervantes, J. E. (2008). Nivel de motivación y uso de estrategias de aprendizaje en alumnos del Programa Educativo Licenciado en Ciencias de la Educación, Itson Empalme. En R. Pizá, M. González y C. Bojórquez (comp.). Estrategias universitarias para mejorar los resultados de enseñanza-aprendizaje (pp. 58-66). México: Itson.

De la Barrera, M. L., Donolo, D. y Rinaudo, M. C. (2010). Estilos de aprendizaje en alumnos universitarios: peculiaridades al momento de aprender. Estilos de Aprendizaje, 6(6), 48-66.

Deci, E. L. (1972). The effects of contingent and noncontingent rewards and controls on intrinsic motivation. Organizational Behaviour and Human Performance, 8(2), 217-229. 
Deci, E. L. y Ryan, R. M. (1985). Intrinsic motivation and self-determination in human behaviour (pp. 1-367). Nueva York: Plemuim.

Donche, V. y Van Petegem, P. (2009). The development of learning patterns of student teachers: A cross-sectional and longitudinal study. Higher Education, 57, 463-475. Dol: 10.1007/s10734-008-9156-y.

Entwistle, N. J. (1988). Motivational factors in students' approaches to learning. In R.R. Schmeck (ed.), Leaming Strategies and Leaming Styles (pp. 21-51). Nueva York: Plenum Press.

Entwistle, N. J. (1991). Approach to learning and perceptions of the learning environment. Introduction to the special issue. Higher Education, 22, 201-204. DOI: 10.1007/BF00132287.

Ferla, J., Valcke, M. y Schuyten, G. (2008). Relationship between student cognitions and their effects on study strategies. Learning and Individual Differences, 18, 271-278. Dol: 10.1016/j.lindif.2007.11.003.

Fernández Sánchez, N. (2011). Promoción del cambio de estilos de aprendizaje y motivaciones en estudiantes de educación superior mediante actividades de trabajo colaborativo en blending learning. Revista Iberoamericana de Educación a Distancia, 14(2), 189-208.

Gaeta González, M. L. (2015). Aspectos personales que favorecen la autorregulación del aprendizaje en la comprensión de textos académicos en estudiantes universitarios. Revista de docencia Universitaria, REDU, 13(2), 17-35.

González, M. L. y Difabio de Anglat, H. E. (2016). Enfoque transversal y longitudinal en el estudio de patrones de aprendizaje en alumnos universitarios de ingeniería. Actualidades Investigativas en Educación, 16(3), 1-20. DOI: http://dx.DOI.ORG/10.15517/AIE.v16I3.26089.

Grajales Grajales, D. M. (2016). La influencia de la autorregulación en la implementación de estrategias de aprendizaje en estudiantes universitarios (tesis de maestría). Universidad Católica de Manizales, Manizales, Colombia.

Granados, H., y Gallego, F. A. (2016). Motivación, aprendizaje autorregulado y estrategias de aprendizaje en estudiantes de tres universidades de Caldas y Risaralda. Revista Latinoamericana de Estudios Educativos, 12(1), 71-90.

Kalaca, S. y Gulpinar, M. (2011). A Turkish study of medical student learning styles. Education for Health Journal, 24(3), 459-469. Recuperado de http://www.educationforhealth.net.

Kashitani, Y. (2005). Las concepciones sobre el aprendizaje a lo largo de la vida de los docentes universitarios. Estudio exploratorio entre 
docentes de la Universidad Iberoamericana, Ciudad de México (tesis de maestría). Universidad Iberoamericana, Ciudad de México.

Marambe, K. N., Athuraliya, T. N., Vermunt, J. D. y Boshuizen, H. P. A. (2007). A comparison of learning strategies, orientations and conceptions of learning of first-year medical students in a traditional and an innovative curriculum. Annals Academy of Medicine, 34, 751-755.

Márquez Jiménez, A. (2017). A 15 años de PISA: resultados y polémicas. Perfiles Educativos, 39(156), 3-15. Recuperado de: http://www.scielo.org.mx/scielo.php?script=sci_arttextypi$\mathrm{d}=$ S0185-26982017000200003ylng=esytlng=es.

Martínez-Fernández, J. R. (2004). Concepción de aprendizaje, metacognición y cambio conceptual en estudiantes universitarios de psicología (tesis doctoral). Universidad de Barcelona, Barcelona, España.

Martínez-Fernández, J. R. y García-Ravidá, L. (2012). Patrones de aprendizaje en estudiantes universitarios del Máster en Educación Secundaria: variables personales y contextuales relacionadas. Profesorado. Revista de Currículum y Formación del Profesorado, 16(1), 165-182.

Marton, F., Dall' Alba, G. y Beaty, E. (1993). Conceptions of learning. International Journal of Educational Research, 19, 277-300.

Morales-Cadena, G. M., Fonseca-Chávez, M. G., Valente-Acosta, B., y Gómez-Sánchez, E. (2017). La importancia de la motivación y las estrategias de aprendizaje en la enseñanza de la medicina. An Orl Mex, 62(2), 97-107.

Parres Soto, R. E., y Flores Macías, R. D. C. (2011) Experiencia educativa en arte visual diseñada bajo un modelo de autorregulación del aprendizaje con estudiantes universitarios. Revista Mexicana de Investigación Educativa, RMIE, 16(49), 597-624.

Pintrich, P. R. (1994). Continuities and discontinuities: Future directions for research in educational psychology. Educational Psychologist, 29, 37-148. DOI: 10.1007/s10648-004-0006-X.

Pintrich, P. y De Groot, E. (1990). Motivational and self-regulated learning components of classroom academic performance. Journal of Educational Psychology, 82, 33-40.

Pintrich, P. R., McKeachi, W., Smith, D., Doljanac, R., Lin, Y., Naveh-Benjamin, M., Crooks, T. y Karabenick, S. (1988). Motivated Strategies for Learning Questionnaire. The University of Michigan (Ncriptal).

Pintrich, P. R., Smith, D. A. F., García, T. y McKeachie, W. J. (1993). Reliability and predictive validity of the Motivated Strategies for Learning Questionnaire (MSLQ). Educational and Psychological Measurement, 53, 801-813. DOI: 10.1177/0013164493053003024. 
Pintrich, P., Smith, D. García, T. y McKeachie, W. (1991). A manual for the use of the Motivated Strategies for Learning Questionnaire (MSLQ). University of Michigan, National Center for Research to Improve Postsecondary Teaching and Learning, Ann Arbor, MI.

Ramírez Dorantes, M. D. C., Canto y Rodríguez, J. E., Bueno Álvarez, J. A., Echazarreta Moreno, A. (2013). Validación psicométrica del Motivated Strategies for Learning Questionnaire en universitarios mexicanos. Electronic Journal of Research in Educational Psychology, 11(1), 193-214.

Rocha, M. y Ventura, M. (2011). Vermunt's learning styles: Searching for Portuguese college student's functioning. Review of Learning Styles, 8(8), 46-70.

Säljö, R. (1979). Learning in the learner's perspective. I: some common-sense conceptions. (Report 76). Sweden: University of Göteborg.

Smith, L., Krass, I., Sainsbury, E. y Grenville, R. (2010). Pharmacy students' approaches to learning in undergraduate and graduate entry programs. American Journal of Pharmaceutical Education, 74(6), 1-6.

Sotelo, M. (2007). Motivación, estrategias de aprendizaje y rendimiento académico en estudiantes universitarios (tesis inédita de maestría). Instituto Tecnológico de Sonora, Sonora, México.

Soto Ortiz, J. L. y Torres Gastelú, C. A. (2016). Percepciones y expectativas del aprendizaje en jóvenes universitarios. Revista de docencia Universitaria, REDU, 14(1), 51-67.

Vázquez, S. (2009). Rendimiento académico y patrones de aprendizaje en estudiantes de ingeniería. Ingeniería y Universidad, 13, 105-136.

Veenman, M. V. J., Prins, F. J. y Verheij, J. (2003). Learning styles: Self-reports versus thinking-aloud measures. British Journal of Educational Psychology, 73, 357-372.

Vermunt, J. D. (1996). Metacognitive, cognitive and affective aspects of learning styles and strategies: A phenomenographic analysis. Higher Education, 31, 25-50.

Vermunt, J. D. (1998). The regulation of constructive learning processes. British Journal of Educational Psychology, 68, 149-171. DOI: 10.1111/ j.2044-8279.1998.tb01281.x.

Vermunt, J. D. (2005). Relations between student learning patterns, personal and contextual factors, and academic performance. Higher Education, 49, 205-234. DOI: 10.1007/s10734-004-6664-2.

Vermunt, J. D., Bronkhorst, L. y Martínez-Fernández, J. R. (2014). The dimensionality in student learning patterns in different cultures. En D. Gijbels, V. Donche, J. Richardson y J. D. Vermunt (eds). Learning Patterns in Higher Education: Dimensions and Research Perspecti- 
ves. New Perspectives on Learning and Instruction, serie. New York: Routledge.

Vermunt, J. D. y Donche, V. (2017). A learning patterns perspective on student learning in higher education: State of the art and moving forward. Educational Psychology Review, 29, 269-299. Dol: 10.1007/ s10648-017-9414-6.

Vermunt, J. D. y Minnaert, A. (2003). Dissonance in students learning pattern: when to revise theory? Studies in Higher Education, 28(1), 49-61. DOI: 10.1080/0307507032000031127.

Weiner, B. (1979). A theory of motivation for some classroom experiences. Journal of Educational Psychology, 71, 3-25. DOI:10.1037/0022-0663.71.1.3.

Weiner, B. (1983). Some thoughts about feelings. En Paris, Olson y Stevenson, Learning and motivation in the classroom (pp. 165-178). Hillsdale, NJ: Erlbaum.

Weinstein, C. E. y Mayer, R. E. (1986). The teaching of learning strategies. En M. Wittrock, Handbook of research of teaching (pp. 315-327). Nueva York: Mcmillan.

Zimmerman, B. J. (1989). A social cognitive view of self-regulated academic learning. Journal of Educational Psychology, 81, 329-339.

Zimmerman, J. (1990). Self-regulated learning and academic achievement: An overview. Educational Psychologist, 25(1), 3-17.

\section{Referencias sobre estudios de patrones de aprendizaje}

De la Garza Pérez, H. J. (2015). Patrones de aprendizaje en estudiantes de Psicología del Norte de México (tesis de maestría). Universidad Autónoma de Nuevo León, México.

Gamboa-Salcedo, T., García-Durán, R. y Peña-Alonso, Y. R. (2012). Traducción al español y análisis de confiabilidad del Inventario de estilos de aprendizaje de Vermunt en residentes de pediatría. Investigación en Educación Médica, 1(2), 57-63.

García-Ravidá, L. B. (2017). Patrones de aprendizaje en estudiantes Latinoamericanos: dimensión cultural e implicaciones educativas (tesis doctoral). Universitat Autònoma de Barcelona, Barcelona, España.

Martínez-Fernández, J. R. y Vermunt, J. D. (2015). A cross-cultural analysis of the patterns of learning and academic performance of Spanish and Latin-American undergraduates. Studies in Higher Education, 40(2), 278-295. DOI:10.1080/03075079.2013.823934. 